\title{
An audit of the dietary intake of Australian children with type 1 diabetes
}

\author{
Heather R. Gilbertson ${ }^{1}$, Kristen Reed ${ }^{2}$, Sarah Clark ${ }^{1}$, Kate L. Francis $\mathbb{1}^{3}$ and Fergus J. Cameron ${ }^{2}$
}

\begin{abstract}
To understand what children with type 1 diabetes in a representative tertiary hospital clinic are eating compared to their peers and explore dietary intake impact on HbA1c outcome. An open cross-sectional dietary audit of children and adolescents with diabetes aged 2-17 years attending the Royal Children's Hospital, Melbourne was conducted using an age-appropriate validated Food Frequency Questionnaire. Total energy, macronutrient intake and diet quality were calculated and compared to dietary advice provided and national intake data. Body weight, and dietary intake influences on glycaemic control were investigated. Overall, 785 patients were recruited, from which 429 dietary surveys were completed. Dietary intakes were overall nutritionally adequate with macronutrient distribution (\% total energy intake) being lower carbohydrate (48.6\%), higher total sugars (22.4\%), fat (32.9\%), saturated fat (14.9\%) and protein intake (19.1\%) than recommendations, but similar to their peers. Energy intakes were excessive compared to their peers in the 4-13 year olds. Rates of overweight (30\%) were significantly higher than national data (18\%). Overall, $43 \%$ achieved optimal glycaemic control ( $\mathrm{HbA1c}<7.5 \%$; $<58 \mathrm{mmol} / \mathrm{mol})$. $\mathrm{HbA} 1 \mathrm{c}$ prediction via linear regression indicated that the following factors were associated with lower $\mathrm{HbA1c}$ values: being male, on pump regimen, lower rates of insulin per $\mathrm{kg}$, shorter duration of disease. This audit has identified areas requiring targeted education/support to improve health outcomes including dietary adherence, rates of overweight/obesity, appropriate energy intakes and optimal glycaemic targets. Furthermore, it provides baseline data to evaluate efficacy of future interventions.
\end{abstract}

\section{Introduction}

Nutritional management is one of the fundamental cornerstones of diabetes management to achieve optimal diabetes control, with $\mathrm{HbA} 1 \mathrm{c}<7.5 \% \quad(<58 \mathrm{mmol} / \mathrm{mol})$ associated with reduced risk of microvascular complications $^{1,2}$. Despite this emphasis on the importance of dietary education and management, the actual dietary intake of Australian children and adolescents with diabetes is not well documented.

Current dietary advice given to children and adolescents with diabetes is based on general healthy eating principles $^{3}$. In Australia these principles are based on the Australian Dietary Guidelines for Children and

\footnotetext{
Correspondence: Heather R. Gilbertson (heather.gilbertson@rch.org.au) 'Department of Nutrition and Food Services, Royal Children's Hospital, Melbourne, VIC, Australia

2Department of Endocrinology and Diabetes, Royal Children's Hospital,

Melbourne, VIC, Australia

Full list of author information is available at the end of the article
}

Adolescents ${ }^{4}$ and is consistent with national ${ }^{5}$ and international $^{6}$ guidelines. At diagnosis, children and their caregivers are provided with two formal diet education sessions. General healthy eating principles are taught with emphasis on eating regular meals and snacks based on low glycaemic index (GI), low fat, low sugar and high fibre food choices. Carbohydrate recommendations are based on $\sim 50 \%$ of total energy intake, with an even distribution over the day, and particular attention given to quantity (carbohydrate counting) and quality (low GI food types).

Adherence to dietary advice is often reported as one of the most difficult aspects of diabetes management as impacts on the social and emotional aspects of eating. Previous studies report poor dietary compliance in American youths whose dietary intake substantially failed to meet national nutrition recommendations ${ }^{7-9}$.

National data for general population intake are available from the 2012 Australian National Nutrition Survey $(\mathrm{NNS})^{10}$, which also combines data from the National 
Health Survey and the National Children's Nutrition and Physical Activity Survey ${ }^{11}$ and are used to compare nutrient intake, physical activity levels and anthropometric measurements.

To date, limited evidence is available regarding compliance to dietary advice in Australian children with diabetes. A cross-sectional audit was undertaken in our clinic to determine the activity and dietary intake of our clinic patients. The information was compared to the dietary advice received and to age-matched National Nutrition Intake data to see how our children with diabetes were faring. With the dietary insight obtained from this audit, it is planned to identify areas of need for targeted nutritional management strategies/resources to improve health outcomes, minimise diabetes-related complications and improve the individual's quality of life.

\section{Subjects and methods}

An open, cross-sectional dietary audit of children and adolescents with diabetes who attended the Diabetes Clinic at the Royal Children's Hospital Melbourne, Victoria was conducted over a six-month period between February-July 2014. Inclusion criteria included aged between 2-17 years inclusive, and diagnosed with diabetes. Exclusion criteria included patients $<2$ years or $>18$ years, and non-English speaking families who are unable to read/write English and therefore were unable to complete the FFQ.

Eligible participants were approached by the research dietitian while waiting for their appointment in clinic and invited to complete two questionnaires. Information statements were provided and written consent completed. All procedures in this study were approved by the Royal Children's Hospital Human Research Ethics Committee (HREC 33214A).

The first questionnaire participants were asked to complete was the Australian Child and Adolescent Eating Survey Food Frequency Questionnaire (ACAES-FFQ). This is a self-administered, semi-quantitative 120-item questionnaire validated for ranking dietary intakes for children and adolescents aged 2-17 years ${ }^{12}$. Reproducibility and comparative validity analyses for this tool has been established $^{12,13}$. The ACAES-FFQ was completed by the child $\geq 12$ years or alternatively by the parent/caregiver on behalf of the child. The ACAES-FFQ was sent to the University of Newcastle for scanning, and nutrient analysis was completed using FoodWorks (Version 3.02.581) to generate individual mean daily macro- and micronutrient intakes. Macronutrient intakes were compared to dietary advice provided and national intake data. Micronutrient intakes were compared to Recommended Daily Intakes $^{14}$.

Data from the ACAES-FFQ were used to calculate an Australian Child and Adolescent Recommended Food
Score (ACARFS) as a measure of overall diet quality, food variety and nutritional adequacy ${ }^{15}$, with a maximum potential score of 73 . Scores $\geq 32$ are considered good diet quality with reasonable diet variety, scores 19-31 indicate moderate diet quality, and scores $\leq 18$ are indicative of poor diet quality with limited diet variety ${ }^{15}$.

The second questionnaire participants were requested to complete was an additional 17-item questionnaire that collected information on socioeconomic status, selfreported physical activity levels, engagement with diabetes healthcare team members, quality of carbohydrate intake and aspects of diabetes management including aspects of insulin adjustment, carbohydrate counting and frequency of self blood glucose monitoring.

A reply paid return envelope was provided to participants unable to complete the questionnaires in the given time, and followed up in their next clinic visit if the questionnaires had not been returned.

Baseline clinical data measures were collected from their recent diabetes visit. This included gender, age, diabetes type, duration of diabetes, relevant co-morbidities, insulin regimen and dosage, most recent $\mathrm{HbA} 1 \mathrm{c} \%$ $(\mathrm{mmol} / \mathrm{mol})$, height $(\mathrm{cm})$ and weight $(\mathrm{kg})$, from which $z$ scores for height, weight and BMI were subsequently calculated. All participant data were de-identified for inclusion in the study.

\section{Data analysis}

Data are reported as mean \pm SD for continuous variables and as proportions for categorical data. Mean BMI $z$-scores were calculated and categorised to allow a continuous version of the BMI score to be used in analysis. For the outcomes of BMI $z$-score and HbA1c value, univariate tests were conducted to determine whether there were differences in the sample based on sex, age and treatment regimen. Further, HbA1c value was predicted via linear regression. Data were analysed using Stata 14.2 (Stata/IC14.2, StataCorp 4905 Lakeway Drive, College Station, Texas 77845, USA).

\section{Results}

\section{Participants}

A total of 785 participants were consented into the study (Fig. 1). For the purpose of this report, only data for those with type 1 diabetes mellitus $(n=765)$ were included in this analysis. Three hundred and seventeen participants did not complete the ACAES-FFQ, and as per the guidelines for using the ACAES-FFQ ${ }^{16}$, participants reporting $>18,000 \mathrm{~kJ} /$ day energy intake $(n=7)$ or with $\geq 5$ incomplete responses $(n=12)$ were excluded. This resulted in a final sample of 429 children with type 1 diabetes reported for this paper (Table 1). Only 6/ 20 subjects with type 2 diabetes reliably completed the 


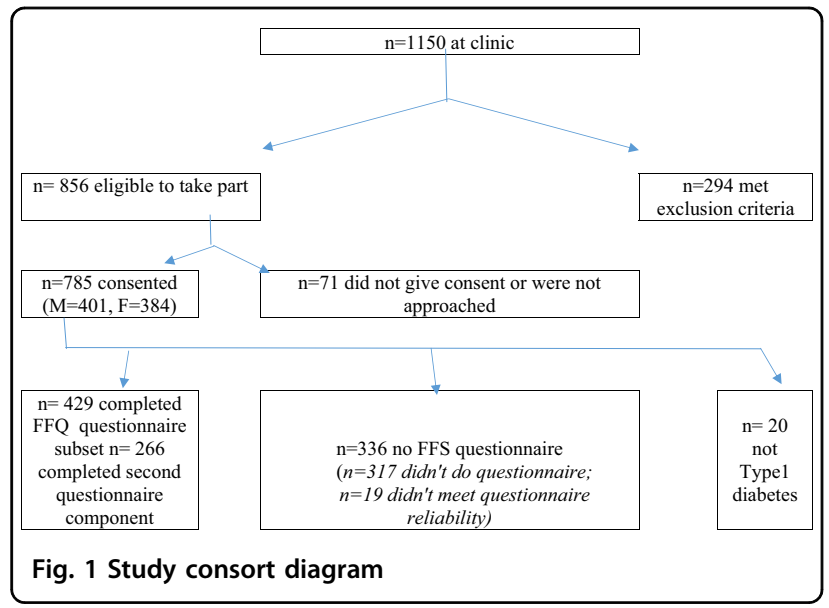

ACAES-FFQ which was considered too small a subset for comparison, hence their exclusion from this analysis.

When those who completed the ACAES-FFQ $(n=429)$ are compared to those who consented but did not complete the questionnaire $(n=317)$, it was found that noncompleters reported having diabetes for a longer duration 62.5 months (95\% CI: 57.8, 67.2) than completers (54.3 months; 95\% CI: 50.4, 58.2, $p=0.008$ ). Noncompleters had higher insulin rate per $\mathrm{kg}$ of 1.05 (95\% CI: $1.01,1.09)$ compared to completers 1.00 (95\% CI: 0.99 , 1.05) $(p=0.033)$, higher HbA1c $8.10 \%(65 \mathrm{mmol} / \mathrm{mol})$ (95\% CI: $7.95,8.24 \%$ or $63,67 \mathrm{mmol} / \mathrm{mol}$ ) compared with $7.88 \%(63 \mathrm{mmol} / \mathrm{mol})(95 \% \mathrm{CI}: 7.76,8.00 \%$ or 61,64 $\mathrm{mmol} / \mathrm{mol})(p=0.020)$ and higher BMI $z$-score $(0.79$ (95\% CI: $0.69,0.89)$ versus 0.64 (95\% CI: $0.56-0.73)(p=$ $0.021)$ ).

\section{Dietary intake and quality}

Mean energy and macronutrient intakes (carbohydrate, sugars, protein, total fat and saturated fat expressed as \% of total energy) were calculated for gender and age groups for comparison to the National Nutrition Survey ${ }^{17}$ (Table 2). Energy intake increased with age from 2 to 13 years as expected. When comparing the current sample to the national sample there was evidence that the total nutrient intake $(\mathrm{kJ})$ was greater in the study sample of 4 to 8 years olds who had a mean intake of $9933 \mathrm{~kJ}(95 \% \mathrm{CI}$ : 9339, 10,526) compared with $7053(p<0.001)$; 9-13 year olds had a mean energy intake of $10159 \mathrm{~kJ}$ (95\% CI: 9755 , $10,563)$ compared with $8603 \mathrm{~kJ}(p<0.001)$. Those aged 14 years or older reported overall lower energy intakes than the national sample $8986 \mathrm{~kJ}$ (95\% CI: 8472, 9500) compared with $9158 \mathrm{~kJ}$; however, there was no evidence that these values were substantially different $(p=0.509)$.

A high proportion of our children across the age groups met $\geq 80 \%$ RDI requirement for age (Table 3) for most micronutrients apart from children aged 2-3 years who were reported to have low intakes of iron and adolescents aged $\geq 14$ years low in folate, calcium, magnesium and iron, comparable to the national data set ${ }^{18}$.

Diet quality as measured by the Australian Dietary Guidelines was also considered. Our data indicated a better daily fruit intake compared to reported National data, but still suboptimal to recommendations. Overall, $78 \%$ of the $2-3$ year olds reported eating the recommended 1 serve per day. This trend decreased with age with $68.5 \%$ of $4-8$ years olds eating 1.5 serves per day (NNS 61\% for this subgroup), 44.9\% of 9-13 year olds eating 2 serves per day, and only $27.8 \%$ of $>14$ years olds eating 2 serves per day (versus only 1\% reported in NNS for this age group).

The mean diet quality ACARFS score of the overall cohort was $30.6 \pm 9.4$, which is representative of moderate diet quality (Table 4). No differences were observed between males $(30.5 \pm 10.0)$ and females $(30.6 \pm 8.7 ; t=$ $-0.06, p=0.956$ ). Diet quality scores were highest for $2-3$ year olds $32.2 \pm 7.9$, similar for $4-8$ year olds $31.7 \pm 9.9$, and 9-13 year olds $32.1 \pm 9.2$, whereas they were lower for those $\geq 14$ years who scored $28.0 \pm 8$.9. Those aged $2-3$ years differed from the other age groups with regard to: having a greater proportion of energy from core foods; and a lower proportion of energy from takeaway food. The individual subcategory ACARFS scores were also compared between each age group which included the ACARFS for intake of vegetables, fruit, meat, protein alternatives, grains, dairy, water and extras (Table 4). All age groups scored well below the total available score within each subcategory, with each score fairly consistent between the different age groups. The impact of insulin treatment modality on nutritional quality was also considered. Insulin treatment modality was recorded as either BD (twice daily injections of mixed insulin), MDI (multiple daily injections of four insulin injections per day using an insulin pen, also known as a basal bolus regime) or Pump (a continuous subcutaneous insulin infusion). In this sample those on BD had mean ACARFS score of $31 \pm$ 10 , MDI $30 \pm 9$, Pump $32 \pm 8$, indicating that those on insulin pump regimens demonstrated greater dietary variety and quality.

There were 266 participant responses on the second questionnaire with regard to food product types, 58\% reported selecting low to moderate glycaemic index (GI) bread types, $66 \%$ reported selecting low to moderate GI cereal types, $71 \%$ reported eating ordinary potato (not the low GI varieties) and 70\% reported eating low GI Basmati rice for those who consumed these food types.

\section{BMI and body weight}

Rates of overweight and obesity were also explored. BMI was calculated and analysed using BMI cut-off points from $\mathrm{Cole}^{19}$ to allow for direct comparison to the National data $\operatorname{set}^{20}$ (Table 5). Our survey reported higher 
Table 1 Demographic and clinical characteristics of the type 1 diabetes patients $(N=429)$

\begin{tabular}{|c|c|}
\hline & $N(\%)$ \\
\hline \multicolumn{2}{|l|}{ Sex } \\
\hline Male & $217(50.6 \%)$ \\
\hline Female & $212(49.4 \%)$ \\
\hline \multicolumn{2}{|l|}{ Age } \\
\hline $2-3$ years & $9(2.1 \%)$ \\
\hline $4-8$ years & $92(21.4 \%)$ \\
\hline 9-13 years & $177(41.3 \%)$ \\
\hline$\geq 14$ years & $151(35.2 \%)$ \\
\hline \multicolumn{2}{|l|}{ Treatment regime } \\
\hline BD (twice daily) & $157(36.6 \%)$ \\
\hline MDI (basal bolus) & $139(32.4 \%)$ \\
\hline Pump $\left(\mathrm{CSI} \|^{\mathrm{a}}\right)$ & $121(28.2 \%)$ \\
\hline Other (combination therapy) & $12(2.8 \%)$ \\
\hline \multicolumn{2}{|l|}{ BMI $z$-score $(n=427)$} \\
\hline Mean (SD) & $0.6(0.9)$ \\
\hline \multicolumn{2}{|l|}{ Hba1c value $(n=410)$} \\
\hline Mean (SD): full sample & $7.9(1.2)$ \\
\hline $2-3$ years & $7.7(0.6)$ \\
\hline $4-8$ years & $7.7(0.9)$ \\
\hline 9--13 years & $7.8(1.0)$ \\
\hline$\geq 14$ years & $8.1(1.5)$ \\
\hline \multicolumn{2}{|c|}{ Duration of diabetes in months $(n=424)$} \\
\hline Mean (SD) & $54.3(40.6)$ \\
\hline \multicolumn{2}{|l|}{ Insulin per kg $(n=419)$} \\
\hline Mean (SD) & $1.0(0.4)$ \\
\hline
\end{tabular}

${ }^{\mathrm{a} C o n t i n u o u s ~ s u b c u t a n e o u s ~ i n s u l i n ~ i n f u s i o n ~}$

proportion of participants in the overweight category and less in the normal category for both sexes across all age groups compared to the national data, and particularly the adolescent age group for boys $12-15$ years and girls $12-17$ years. When examining BMI $z$-score there was no evidence of difference between males and females $(p=0.073$; $95 \%$ CI: $0.19,0.14)$ or age groups $(p=0.676 ; 95 \% \mathrm{CI}$ : $-0.02,0.01$ ). There was weak evidence that the MDI (BMI $z$-score $=0.80)$ treatment regimen was associated with higher BMI score compared with $\mathrm{BD}(\mathrm{BMI} z$-score $=0.59$; difference of 0.21 (95\% CI: 0.004, 0.42)) and pump (BMI $z$ score $=0.51$; difference of 0.29 (95\% CI: 0.08, 0.49)).

\section{Physical activity level (PAL)}

Self-reported PAL was collected in questionnaire two and was completed by 266 participants. With this consideration in mind, this subgroup reported an average of $82 \mathrm{~min} /$ day which meets the national recommendation of $\geq 60 \mathrm{~min}$ of physical activity every day ${ }^{21,22}$. Overall, $62 \%$ of the cohort met this guideline as compared to $69 \%$ in the NNS. The mean time of physical activity was calculated for each participant using the sum of all self-reported physical activity (at school, outside of school and during leisure) divided by 7 for a daily total. There was no evidence that minutes of PAL was different between males (78.5 $\mathrm{min} /$ day) and females ( $85 \mathrm{~min} /$ day) $(p=0.305 ; 95 \%$ CI: $21.7,6.84)$. Rates of reported activity were inversely related to age with $71 \%$ of $2-3$ year olds, $61 \%$ of $4-13$ year olds, and $62 \%$ of those $\geq 14$ years meeting activity recommendations $\geq 60 \mathrm{~min} /$ day. There was no evidence that reported PAL (minutes per day) was associated with BMI $z$-score $(p=0.431 ; 95 \%$ CI: $-4.9,11.5)$ or age $(p=$ 0.952 95\% CI: $-1.2,1.1)$.

\section{Glycaemic control}

Glycaemic control was evaluated using the most recent HbA1c measure. Mean HbA1c of this cohort was $7.9 \pm$ $0.9 \%(63 \pm 10 \mathrm{mmol} / \mathrm{mol})$ which is consistent with the overall clinic average at that time. Cut-offs were used to determine the proportion of the cohort meeting best practice guidelines of an $\mathrm{HbA} 1 \mathrm{c} \leq 7.5 \%(<58 \mathrm{mmol} / \mathrm{mol})^{5}$, ${ }^{6}$. Categories included optimal control HbA1c $\leq 7.5 \%(<58$ $\mathrm{mmol} / \mathrm{mol}$ ); moderate control $>7.5 \%$ and $\leq 8.5 \%$ ( $>58$ $\mathrm{mmol} / \mathrm{mol}$ and $\leq 69 \mathrm{mmol} / \mathrm{mol}$ ); and poor control HbA1c $>8.5 \%$ (>69 mmol $/ \mathrm{mol}$ ). In our cohort, $43.4 \%$ had optimal glycaemic control, $31.5 \%$ moderate control and $25.1 \%$ poor control. Comparison of treatment regimen found that $54 \%$ of those on pump had optimal control, while $40 \%$ of those with BD and MDI had optimal control. There was evidence that being female compared to male was associated with higher $\mathrm{HbA1c}$ value (group difference of $-0.25 \%$ (95\% CI: $-0.49,-0.02 \%$ ), $t=2.1, p=0.036$ ). Increasing age was associated with $0.03 \%$ (95\% CI: 0.01 , $0.05)$ increase in $\mathrm{HbA1c}(p=0.008)$.

\section{Predicting HBA1C}

A series of univariate tests were run to determine which factors may account for variance in HbA1c including sex, age, insulin regimen, BMI $z$-score, insulin rate per $\mathrm{kg}$, duration of diabetes, ACARFS score, percent of diet from protein/carbohydrate/fats/saturated fats and from 'core foods'. (PAL was not included as only $60 \%$ of respondents completed questionnaire two.) Variables that were significant predictors after univariate testing were included in the model as independent variables (sex, age, insulin regimen, insulin rate per $\mathrm{kg}$, duration of diabetes, ACARFS score, percent of dietary energy total fat intake). The model explained $15 \%$ of the variance in the data. When all variables are held equal the following variables are important predictors of HbA1c values: being female is 


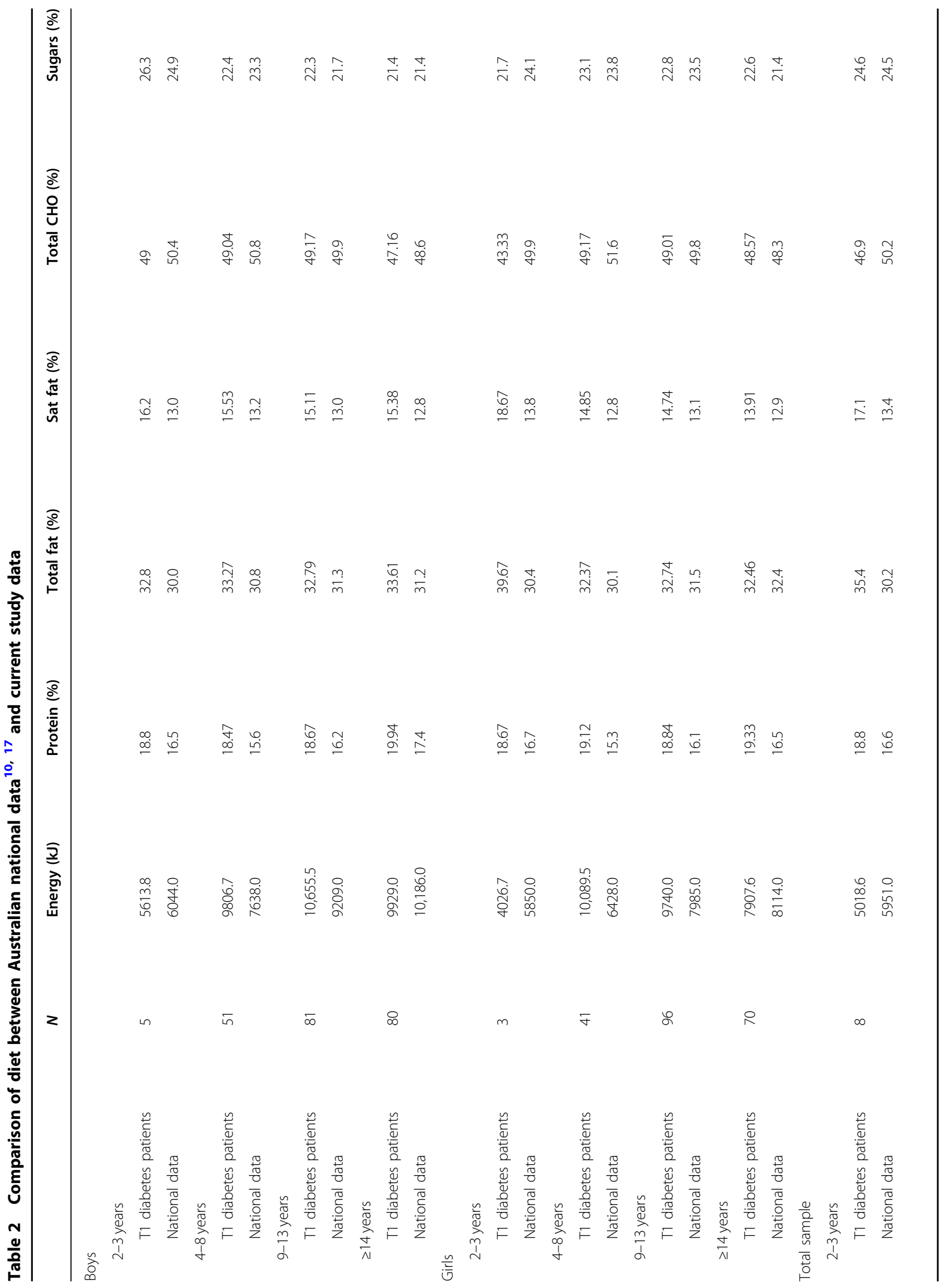




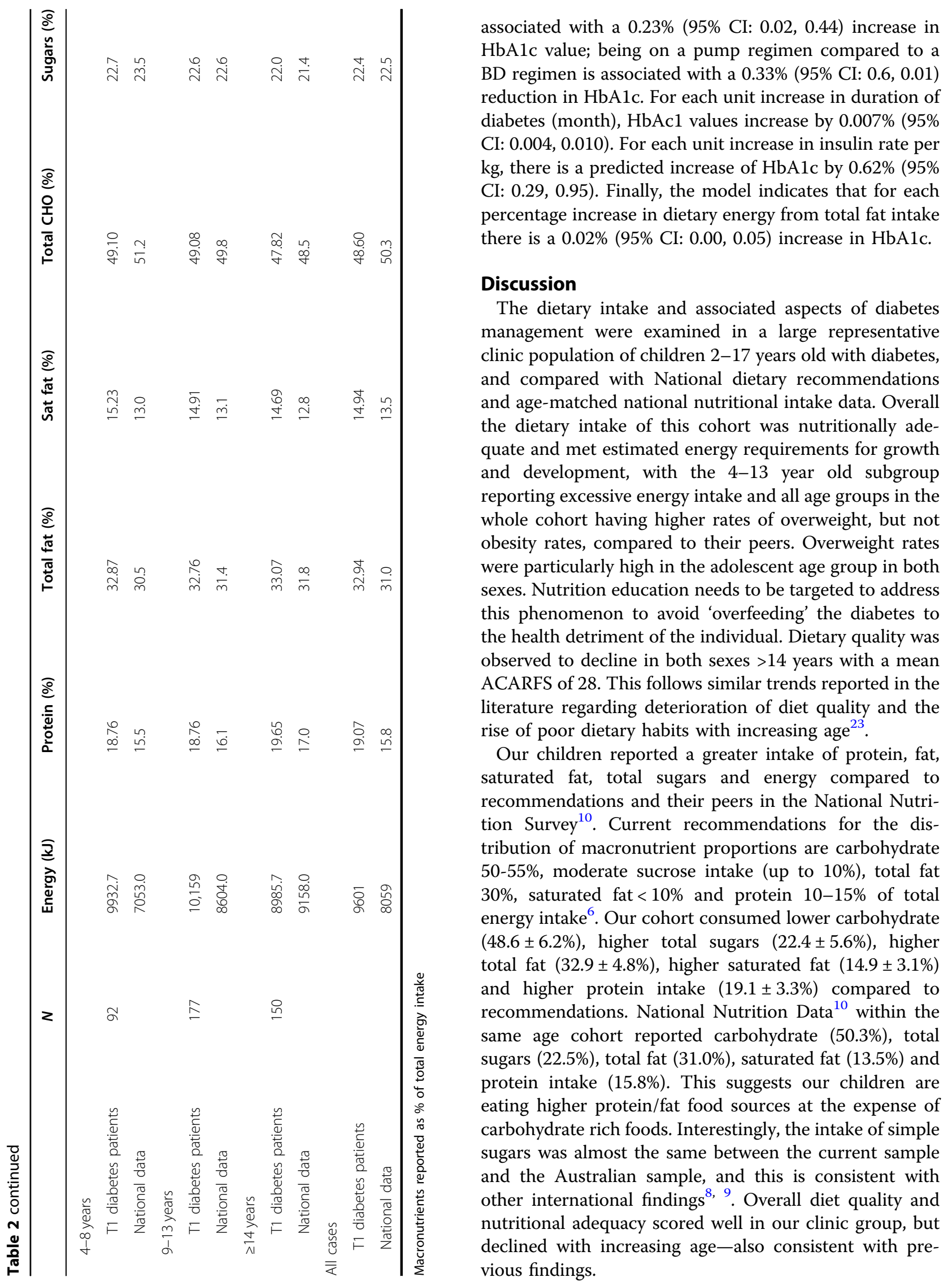




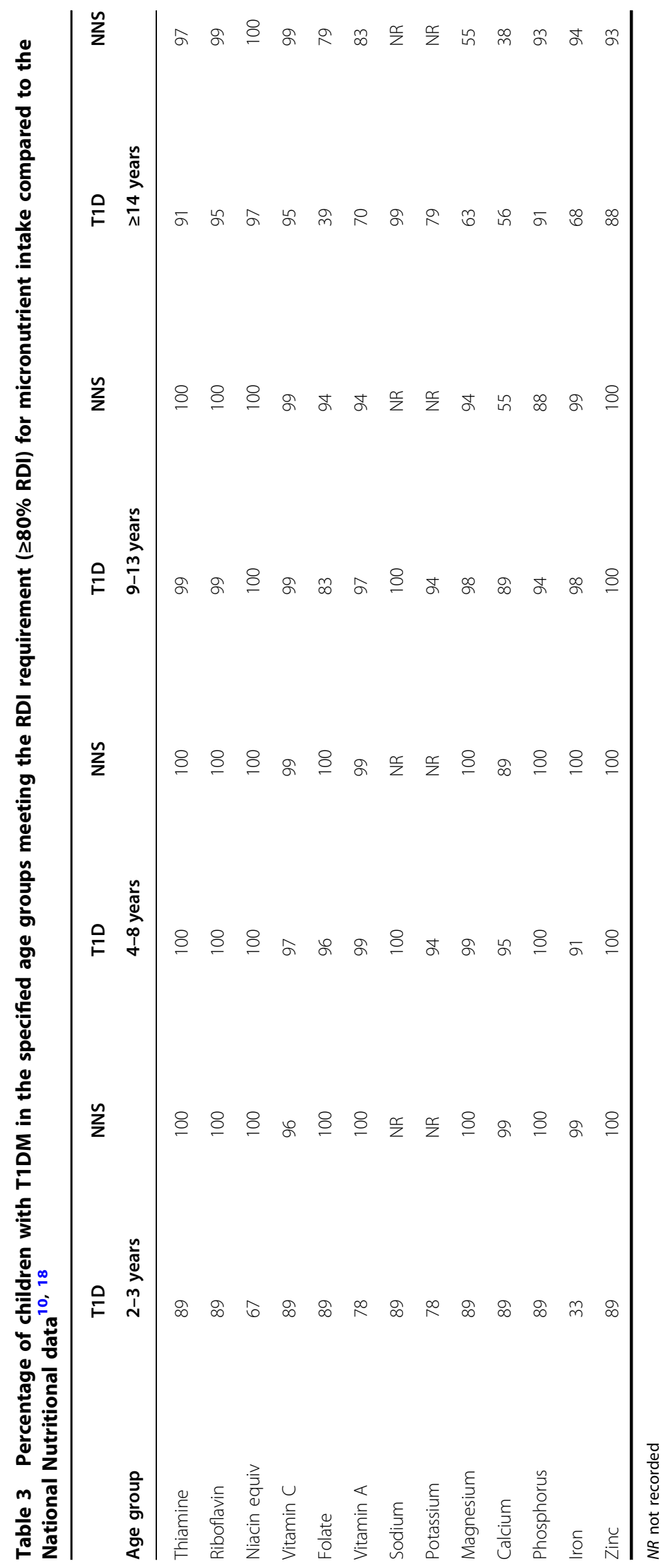




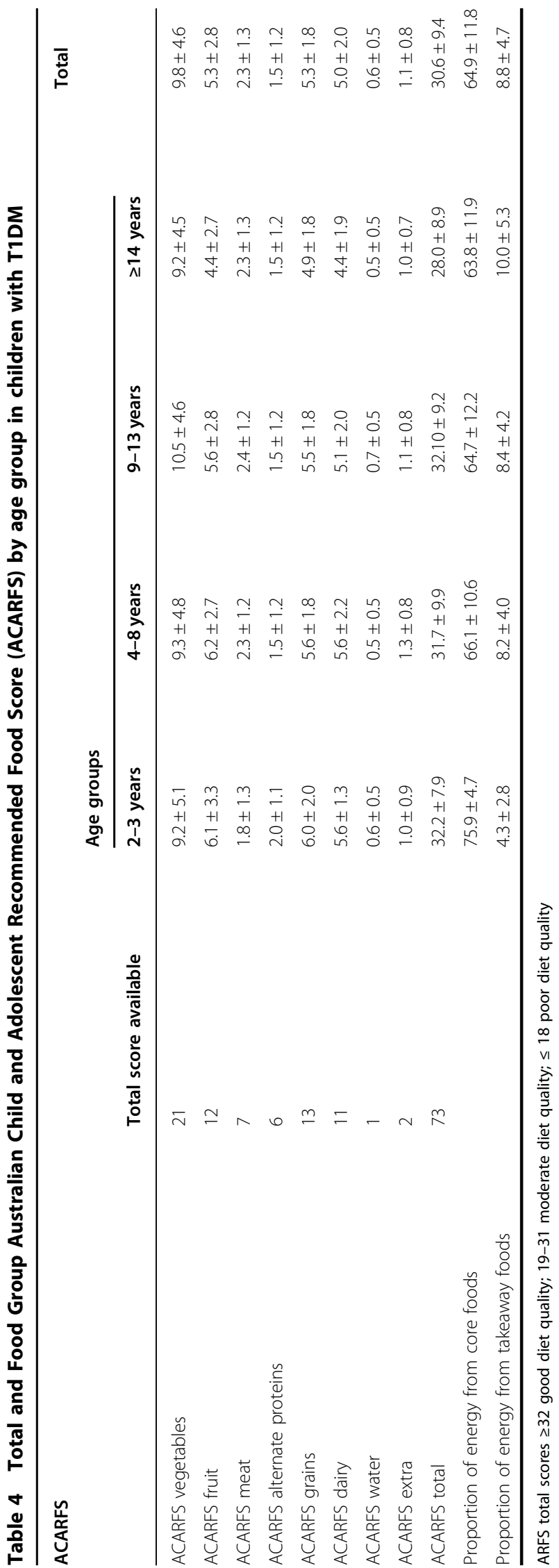

Participants were more overweight than their peers, with increased rates of overweight consistently being reported in other international diabetes centres. ${ }^{24-28}$. Although it is recognised that there is an increasing prevalence of overweight and obesity in the general population $^{29}$, the increased reported rates of overweight in children with diabetes is concerning. Potentially too much emphasis is placed on daily glycaemic targets and increasing insulin doses to improve control and not enough focus on improving the quantity and quality of foods consumed. With increased awareness now of this association, dietetic interventions in the clinic setting can focus more specifically on this issue. Other clinicians in the multidisciplinary team may also be mindful of this inter-relationship before increasing insulin doses. The Diabetes Control and Complications Trial (DCCT) identified weight gain as an adverse consequence of intensive insulin therapy ${ }^{1}$. However, emerging evidence has shown improved glycaemic control can be achieved without inducing weight gain, through careful redistribution of insulin doses to more closely match requirements ${ }^{30}$. Children with diabetes can avoid substantial weight gain by optimising insulin management, adhering to individually tailored nutrition advice and regular participation in physical activities.

Only $43 \%$ of the cohort met the target for good glycaemic control which clearly needs to be addressed. The majority had optimal to moderate glycaemic control however this declined with the transition into adolescence, alongside diet quality. This places the adolescent population at increased risk of having nutritionally inadequate intakes, and has significant implications for the optimisation of their insulin therapy and overall diabetes treatment. Insulin pump therapy was associated with $0.33 \%$ improvement in HbA1c outcomes, however it should be noted that there may be bias in our clinic cohort as it tends to favour the highly motivated and better controlled individuals being considered for this treatment option.

The food frequency questionnaire is regarded as the most practical and economical dietary assessment method for the collection of comprehensive data in larger studies $^{31}$. Issues for consideration when making comparisons with National Nutrition data include the different dietary methodology used for each survey (frequency versus 24-h recall and 3-day food records). Under-reporting is also likely in females $\geq 14$ years as evidenced by our groups lower mean energy intake. Methodology was used to try and identify and exclude the over-reporters. Assessment of dietary intake presents unique challenges and current research suggests there is no single perfect method of dietary assessment as all methods are fraught with the potential of individual under- and over-reporting of intake $^{31,32}$. 
Gilbertson et al. Nutrition and Diabetes (2018)8:10

Page 9 of 12

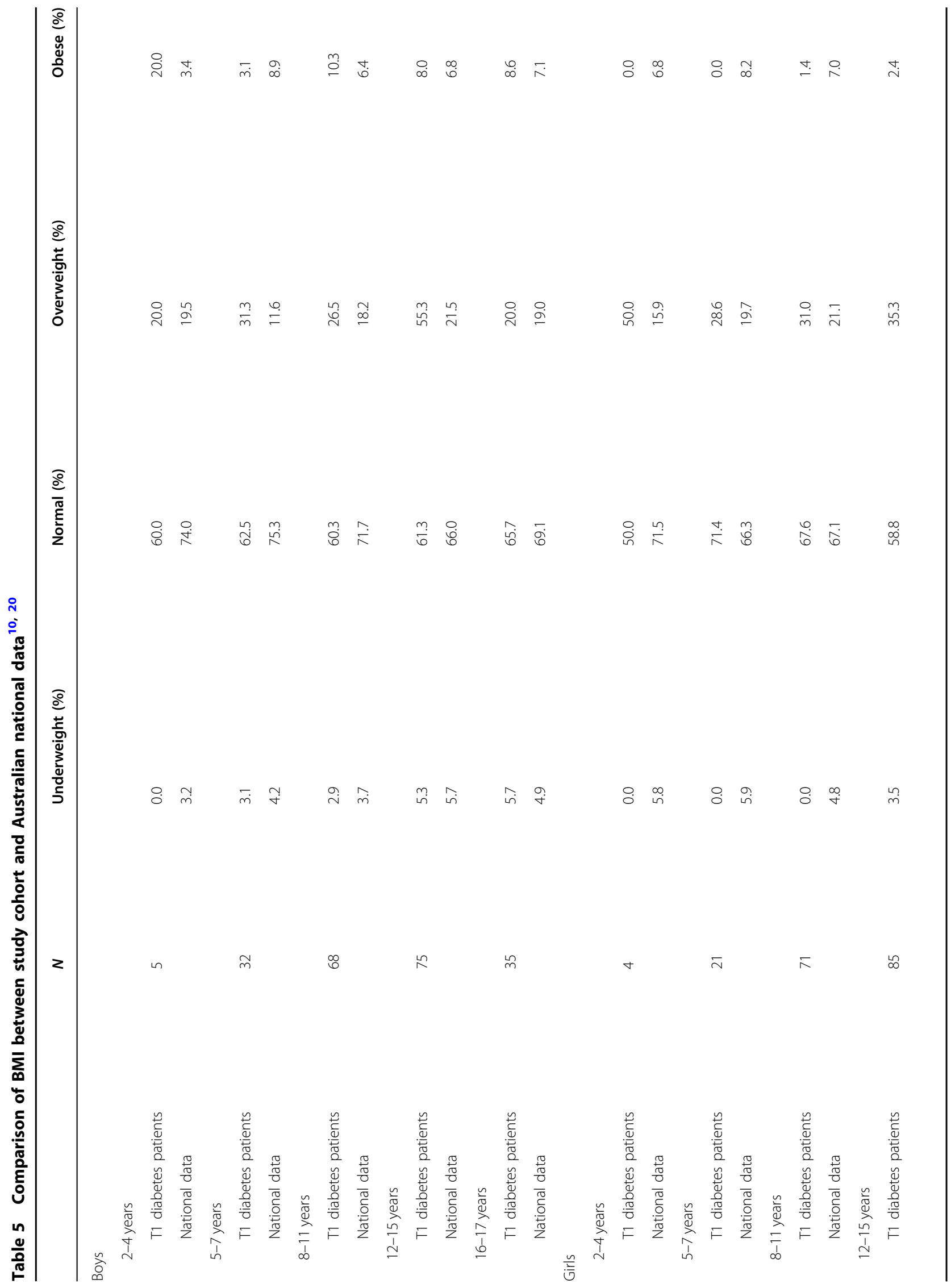

Nutrition and Diabetes 
Gilbertson et al. Nutrition and Diabetes (2018)8:10

Page 10 of 12

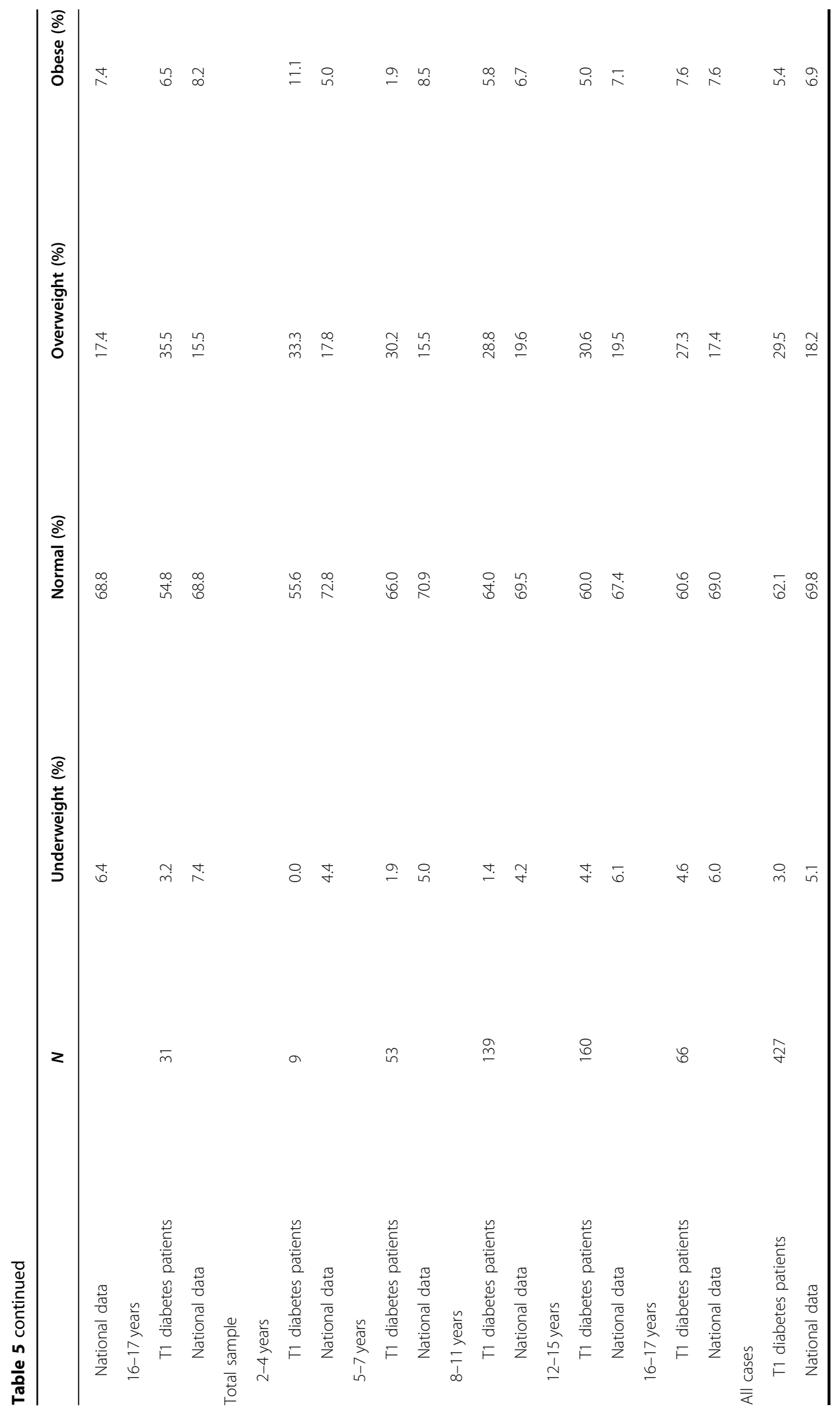

Nutrition and Diabetes 
This audit provides good baseline data for comparison of future targeted nutrition intervention and engagement strategies for our cohort to evaluate efficacy. A comprehensive, multidisciplinary approach to diabetes management is encouraged and this audit highlights the need to consider opportunities to review/expand service delivery models to improve engagement with team members and strive towards strategies to improve overall diabetes control. Dietitians must focus on weight management, specifically reducing dietary fat intake and improving the nutritional adequacy overall, with targeted intensive education to the 4-13 years old group to prevent excessive energy intake that is likely to be detrimental to diabetes-related health outcomes later in life. Additional education and support is required throughout adolescence to attempt to attenuate the decline in nutritional adequacy, diet quality, glycaemic control that is currently occurring with age, to minimise diabetes-related complications and improve quality of life outcomes. Dietary management for children of all ages with diabetes must always consider the medical management and be individually tailored to the highly variable and constantly changing nutritional needs of the growing child.

\section{Acknowledgements}

We thank the student dietitians Sarah Brenner and Patrice Ventura who assisted with data collection and reporting during their placement. We also thank all the patients and their families who participated in this audit.

\section{Author details}

'Department of Nutrition and Food Services, Royal Children's Hospital, Melbourne, VIC, Australia. 'Department of Endocrinology and Diabetes, Royal Children's Hospital, Melbourne, VIC, Australia. ${ }^{3}$ Clinical Epidemiology \& Biostatistics Unit, Murdoch Children's Research Institute, Melbourne, VIC 3052, Australia

\section{Authors' contributions}

H.G. is the guarantor for the project and manuscript. H.G. designed the project, collected and analysed the data, and wrote the manuscript. K.R. collected data, contributed to the discussion and reviewed/edited the manuscript. S.C. collected and analysed data, contributed to the discussion and reviewed/ edited the manuscript. K.F. analysed the data contributed to writing methods, results and reviewed/edited manuscript. F.C. contributed to the discussion and reviewed/edited the manuscript.

\section{Conflict of interest}

The authors declare that they have no conflict of interest.

\section{Publisher's note}

Springer Nature remains neutral with regard to jurisdictional claims in published maps and institutional affiliations.

Received: 27 February 2017 Revised: 19 December 2017 Accepted: 5 February 2018

Published online: 09 March 2018

\footnotetext{
References

1. DCCT Research Group. The effects of intensive diabetes treatment on the development and progression of long-term complications in insulin-
}

dependent diabetes mellitus. The Diabetes Control and Complications Trial. N. Engl. J. Med. 329, 977-986 (1993).

2. DCCT Research Group. The effect of intensive diabetes treatment on the development and progression of long-term complications in adolescents with insulin-dependent diabetes mellitus: The Diabetes Control and Complications Trial. J. Pediatr. 125, 177-188 (1994).

3. Barclay, A., Gilbertson, H., Marsh, K. \& Smart, C. Dietary management in diabetes. Aust. Fam. Physician 39, 579-583 (2010).

4. National Health and Medical Research Council. Australian Dietary Guidelines (National Health and Medical Research Council, Canberra, 2013).

5. Craig, M. E. et al. National Evidence-Based Clinical Care Guidelines for Type 1 Diabetes in Children, Adolescents and Adults (Australian Government Department of Health and Ageing, Canberra, 2011).

6. Smart, C., Aslander-van Vliet, E. \& Waldron, S. Nutritional management in children and adolescents with diabetes. Pediatr. Diabetes 10(Suppl. 12), 100-117 (2009).

7. Randecker, G. A. et al. The dietary intake of children with IDDM. Diabetes Care 19, 1370-1374 (1996)

8. Mayer Davis, E. J. et al. Dietary intake among youth with diabetes: The SEARCH for Diabetes in Youth Study. J. Am. Diet. Assoc. 106, 689-697 (2006).

9. Delahanty, L. et al. Self-reported dietary intake of youth with recent onset of type 2 diabetes: results from the TODAY Study. J. Acad. Nutr. Diet. 113, 431-439 (2013).

10. Department of Health and Ageing. Australian National Children's Nutrition and Physical Activity Survey: Main Findings (Commonwealth of Australia, Canberra, 2012).

11. Department of Health and Ageing. Australian National Children's Nutrition and Physical Activity Survey: Main Findings (Commonwealth of Australia, Canberra, 2007).

12. Watson, J. F., Collins, C. E., Sibbritt, D. W., Dibley, M. J. \& Garg, M. L. Reproducibility and comparative validity of a food frequency questionnaire for Australian children and adolescents. Int J. Behav. Nutr. Phys. Act. 6, 62 (2009).

13. Collins, C. et al. Validation of an Adult Food Frequency Questionnaire and Development of Diet Quality Score for Children and Adults (2011The University of Newcastle, Callaghan, 2011).

14. National Health and Medical Research Council. Nutrient Reference Values for Australia and New Zealand, NHMRC (Commonwealth of Australia, Canberra, 2006).

15. Marshall, S., Watson, J., Burrows, T., Guest, M. \& Collins, C. E. The development and evaluation of the Australian child and adolescent recommended food score: a cross-sectional study. Nutr. J. 11, 96 (2012).

16. Watson J., Collins C., SibbrittDW, Garg M. L., \& Dibley M. J. The Australian Eating Surveys: Food Frequency Questionnaires for Pre-schoolers, Children, Adolescents and Adults User Manual Version 1-April 2013 1-63 (University of Newcastle, Callaghan).

17. Australian Bureau of Statistics. Australian Health Survey: Nutrition First Results-Foods and Nutrients, 2011-12, Australia. Mean Contribution to Energy Intake: Protein, Fat, Carbohydrate, Dietary Fibre and Alcohol Data (Commonwealth of Australia, Canberra, 2014).

18. Australian Bureau of Statistics. Australian Health Survey: Nutrition First Results-Foods and Nutrients, 2011-12, Australia. Mean Daily Energy and Nutrient Intake Data (Commonwealth of Australia, Canberra, 2014).

19. Cole, T. J. \& Lobstein, T. Extended international (IOTF) body mass index cut-offs for thinness, overweight and obesity. Pediatr. Obes. 7, 284-294 (2012).

20. Australian Bureau of Statistics. Australian Health Survey: Updated Results, 2011-12 Body Mass Index Data (Commonwealth of Australia, Canberra, 2013).

21. Department of Health and Ageing. Australia's Physical Activity Recommendations for 5-12 Year Olds (Commonwealth of Australia, Canberra, 2004).

22. Department of Health and Ageing. Australia's Physical Activity Recommendations for 12-18 Year Olds (Commonwealth of Australia, Canberra, 2004).

23. Liu, L. L. et al. Prevalence of overweight and obesity in youth with diabetes in USA: the SEARCH for Diabetes in Youth Study. Pediatr. Diabetes 11, 4-11 (2010).

24. Ferrante, E. et al. Nutritional status, obesity and metabolic control in children with type 1 diabetes mellitus. Minerva Pediatr. 51, 39-46 (1999).

25. Knerr, l. et al. The 'accelerator hypothesis': relationship between weight, height, body mass index and age at diagnosis in a large cohort of 9,248 German and Austrian children with type 1 diabetes mellitus. Diabetologia 48, 2501-2504 (2005). 
26. Libman, I. M., Pietropaolo, M., Arslanian, S. A., Laporte, R. E. \& Becker, D. J. Changing prevalence of overweight children and adolescents at onset of insulin treated diabetes. Diabetes Care 26, 2871-2875 (2003).

27. Reinehr, $T$. et al. Insulin resistance in children and adolescents with type 1 diabetes mellitus: relation to obesity. Pediatr. Diabetes 6, 5-12 (2005).

28. Australian Institute of Health and Welfare (2013). Overweight and obesity. Australian Institute of Health and Welfare. http://www.aihw.gov.au/ overweight-and-obesity/. Accessed September 2014 (2013).

29. Brown, R. J., Wijewickrama, R. C., Harlan, D. M. \& Rother, K. I. Uncoupling intensive insulin therapy from weight gain and hypoglycemia in type 1 diabetes. Diabetes Technol. Ther. 13, 457-460 (2011).
30. Barrett-Connor, E. Nutrition epidemiology: how do we know what they ate? Am. J. Clin. Nutr. 54(Supp 1), 182S-187S (1991).

31. McPherson, R. S., Feaganes, J. R. \& Siegler, I. C. Measurement of dietary intake in the UNC Alumni Heart Study. University of North Carolina. Prev. Med. 31 56-67 (2000).

32. Amed, S. et al. Adherence to clinical practice guidelines in the management of children, youth and young adults with type 1 diabetes: a Prospective Population Cohort Study. J. Pediatr. 163, 543-548 (2013). 